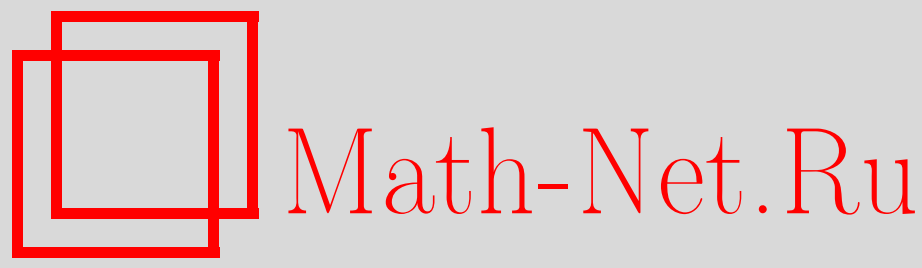

В. В. Юдин, П. Л. Титов, А. Н. Михалюк, Энтропийная мера характера порядка-беспорядка решеточных систем в представлении координационных древесных графов Кэли, ТМФ, 2010, том 164, номер 1, 88-107

DOI: https://doi.org/10.4213/tmf6526

Использование Общероссийского математического портала Math-Net.Ru подразумевает, что вы прочитали и согласны с пользовательским соглашением http://www.mathnet.ru/rus/agreement

Параметры загрузки:

IP : 54.166 .219 .16

26 апреля 2023 г., $11: 44: 38$

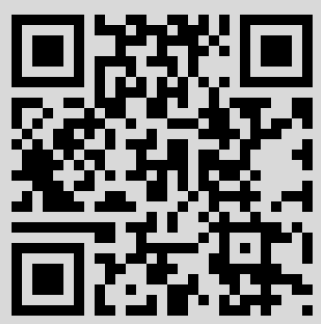




\title{
ЭНТРОПИЙНАЯ МЕРА ХАРАКТЕРА ПОРЯДКА-БЕСПОРЯДКА РЕШЕТОЧНЫХ СИСТЕМ В ПРЕДСТАВЛЕНИИ КООРДИНАЦИОННЫХ ДРЕВЕСНЫХ ГРАФОВ КЭЛИ
}

\begin{abstract}
Приведено систематическое изложение информодинамического метода анализа решеточных и сеточных систем. Установлена логика и алгоритм отображения указанных объектов в координационные древесные графы Кэли, изложены их основные свойства. Древесные графы сеточных систем являются сложными объектами, для изучения которых можно применять принцип симплициальной декомпозиции кустового типа. На основе симплициальной декомпозиции построены перечисляющие структуры, от которых образуются функционалы энтропийного вида. Поставлена задача перколяции на древесных графах Кэли в нетрадиционном понимании, которая может быть рассмотрена как на уровне перечисляющих структур, так и для их энтропий. Соответствующие энтропийные перколяционные зависимости и их критические индексы могут служить достаточно универсальными мерами упорядочения решеточных систем. Свойство симплициальности подразумевает также аналогию с принципом фрактальности. Введены три типа фрактальных характеристик и даны аналитические выражения фрактальных размерностей для тангенциального и стримерного представлений, а также для скорлуп Мандельброта.
\end{abstract}

Ключевые слова: обобщенная решеточная система, древесный координационный граф Кэли, симплициальная декомпозиция, энтропия Вайда, дивергенция Бонгарда, дальний порядок, фрактальная размерность, сверхразмерная перколяция.

\section{1. ВВЕДЕНИЕ}

В классических монографиях [1], [2], а также в монографиях последних годов [3], [4] для изучения проблемы порядка-беспорядка применяется большое число различных показателей ближнего, среднего, дальнего упорядочения. Появились интересные исследования [5], [6] по обобщению метода Вороного-Делоне при исследовании структуры некристаллических систем - аморфных сред, кварцевых и металлических

* Дальневосточный государственный университет, Владивосток, Россия. E-mail: mih-alexey@yandex.ru 
стекол, а также квазикристаллических систем [7], [8]. Одним из широко распространенных методов анализа упорядочения является корреляционно-спектральный метод. Если иметь в виду формулировку критерия дальнего упорядочения в этих терминах, то можно говорить об остаточных корреляциях или потребовать, чтобы огибающая корреляционной функции имела гиперболическое убывание при нулевой асимптотике. Для описания такого поведения удобно ввести критические индексы $\gamma$. Тогда условие дальнодействия в нулевой корреляционной асимптотике сведется к требованию $\gamma \leqslant 1$. В своих ранних работах [9], [10] мы столкнулись с неизвестным ранее феноменом, когда в разупорядоченных неравновесных средах возникает многомасштабная иерархия пространственных неоднородностей. Удалось показать, что при таких условиях дальний порядок реализуется сеточной иерархией естественных неоднородностей мезомасштабного уровня [10]. С математической точки зрения корреляционно-спектральный подход применяется к ансамблю фрагментов (отдельностей), а корреляция призвана учесть взаимосвязи между отдельностями, ячейками и т.д.

Однако наряду с алфавитом отдельностей как структурных единиц главным в сеточных системах является понятие соседства (смежности, инциденции) [11]. При этом сеточные и решеточные системы удобно представлять координационными древесными графами Кэли (ДГК), которые были нами систематически изучены в ряде работ [11]-[15].

\section{2. ИНФОРМОДИНАМИЧЕСКИЙ МЕТОД ОПИСАНИЯ СТРУКТУРЫ РЕШЕТОЧНЫХ И СЕТОЧНЫХ СИСТЕМ}

Пусть нам даны некоторые сеточные и решеточные структуры. По своей топологии они могут обладать различной упорядоченностью. Для удобства будем рассматривать черно-белые сетки, полученные из реальных наноструктурных объектов способом клиппирования. Как правило, сеточные структуры составлены из полигональных выпуклых фрагментов (см. рис. 1a, 2a). Основная задача состоит в создании универсального метода изучения сеточных и решеточных систем. Мы построим адекватное представление сеточных и решеточных структур соответствующими математическими объектами. Эта задача решается в терминах полного взаимно однозначного отображения подобных систем в соответствующие объекты. Наряду с этой задачей необходимо разработать формализм, позволяющий отслеживать динамику и кинетику сеточных и решеточных систем при различных воздействиях, например процессы структурной релаксации, деградации структур, а также консервативного старения [16], [17]. Эти процессы также должны быть описаны в терминах эволюции вышеупомянутых математических объектов [18].

Стандартный способ исследования подобных структур базируется на теории ансамблей, которая фактически относится к статистической физике, теории вероятностей и математической статистике. Если следовать этой методологии, то нужно "разрезать" по границам сетки и решетки и в полученной "россыпи” заниматься 


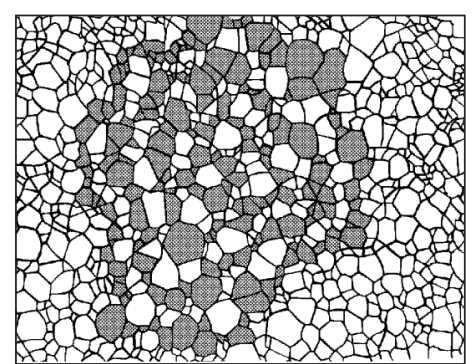

a

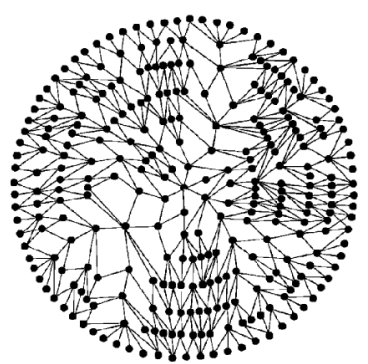

б

Рис. 1. Клипированное изображение для кварцевого стекла КУВИ-1 (а), его древесный граф Кэли (б).

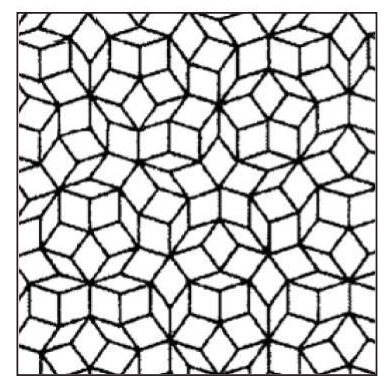

a

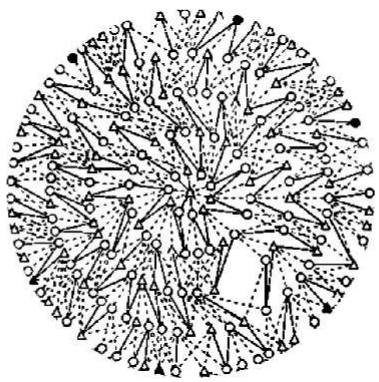

б

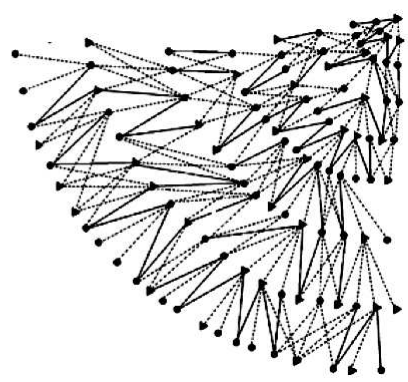

B

Рис. 2. Мозаика Пенроуза (а), ее координационный древесный граф Кэли (б) и его увеличенный фрагмент (в).

статистическим “лото". Как обычно в подобных случаях, ищется функция распределения таких ячеек по размерам (площадям). Для "быстрых" процедур, используя концепцию выпуклых неправильных полигонов, мы базируемся на понятии диаметра этих элементарных единиц. В этом случае мы получаем верхнюю оценку распределения ячеек (зерен) по размерам. Но все же это не сеточная система. Здесь необходимо упомянуть корреляционно-спектральные методы представления и изучения таких сеточных и решеточных систем, которые были реализованы нами в работе [9].

Критикуя ансамблевый метод представления сеточных и решеточных систем, можно указать на потерю некоторой целостности, которая как раз и составляет сущность таких структур. В философии есть термин эмерджентность. Именно этого в корреляционных методах исследования ансамбля зерен, ячеек не существует по определению. Необходимо разработать такой тип представления, который исполнял бы эмерджентные функции. Основой таких функций, по нашему мнению, является понятие инциденции (соседства, координации) ближайших элементарных единиц. Именно инциденции, по нашему мнению, как раз и создают сеточные, решеточные структуры. Поэтому основным аспектом построения адекватного представле- 
ния сеточных, решеточных систем мы считаем учет именно отношения инциденций, смежностей. В определенной мере их даже нужно вынести на первый план. Чтобы представление было адекватным, необходимо сохранить и ячеечную, зеренную компоненту. По нашему мнению, в наибольшей степени этому соответствуют обобщенные древесные графы Бете-Кэли, показанные на рис. 1б, 2б, 2в. Заметим, что это именно координационные графы, которые наиболее непосредственным образом показывают картину связей между ячейками, модулями, зернами. Необходимо отметить, что такие ДГК не являются графами Бете и вообе древесными графами типа "леса" [19], [20].

\section{3. ЛОГИЧЕСКИЕ АСПЕКТЫ ПОСТРОЕНИЯ КООРДИНАЦИОННЫХ ДРЕВЕСНЫХ ГРАФОВ КЭЛИ}

Прежде всего, сформулируем алгоритм отображения сеточных, решеточных структур в такие ДГК и сопроводим процедуру построения логическими и алгебраическими параллелями. Выбираем за центр некоторую пробную ячейку сетки или элементарную плитку мозаики. От нее строим, используя отношение инциденции, первую координационную звезду. Это можно описать следующим простым выражением:

$$
a_{0} \rho^{*}\left(\bigcup^{s_{1}} a_{j_{k_{1}}}\right),
$$

где $a_{0}$ - стартовая вершина графа; $\rho$ - операция инциденции бинарного типа, она устанавливает координационное отношение между стартовой вершиной и некоторым подмножеством вершин следующего уровня $a_{j_{k_{i}}}$, которые являются инцидентно достижимыми ( $\rho^{*}-$ инциденции центральной “звезды”, $\rho^{\downarrow}-$ инциденции последующих уровней, направленные вперед); $s_{i}$ - число вершин $i$-го уровня или общее координационное число.

Сам оператор инциденции подчиняется условию симметричности, не является транзитивным и, как мы увидим ниже, обладает некоторым аналогом свойства ассоциативности, который характеризуется цепной логикой формирования ДГК. От каждой инцидентно достигнутой вершины звезды строятся координационные кусты, направленные только вперед. Связи между элементами одного уровня, а также ветви, направленные назад, запрещены. Тем самым получаем простой двудольный граф в приближении исходящих ветвей, связей. Знак объединения можно трактовать как объединение подмножеств кустового типа на каждом фиксированном уровне. Теперь мы получаем следующее выражение:

$$
a_{0} \rho^{*}\left[\left(\bigcup^{s_{1}} a_{j_{k_{1}}}\right)\right] \cdot \rho^{\downarrow}\left(\bigcup^{s_{2}} a_{j_{k_{2}}}\right) .
$$

В этом выражении начинает формироваться цепочная логика операторов инциденций с их действием на некоторые кустовые подмножества вершин графа. В знаках объединения всегда стоят общие координационные числа $s_{1}<s_{2}<\cdots<s_{i}$. 
Третья и дальнейшие сферы инциденций строятся итеративно по вышеупомянутому правилу (1), (2). Таким образом мы получаем каскад с иерархией двудольных графов. В результате образуется $i$-дольный граф, где $i$ - номера уровней ДГК, $i \rightarrow \infty$. Последнее означает, что мы имеем дело с актуализированным ДГК, и этот этап можно сопроводить следующей итерацией инциденций:

$$
a_{0} \rho^{*}\left[\left(\bigcup^{s_{1}} a_{j_{k_{1}}}\right)\right] \cdot \rho^{\downarrow}\left[\left(\bigcup^{s_{2}} a_{j_{k_{2}}}\right)\right] \cdot \ldots \cdot \rho^{\downarrow}\left[\left(\bigcup^{s_{i}} a_{j_{k_{i}}}\right)\right] \cdot \ldots \cdot
$$

где $j_{k_{i}}$ - внутренний индекс каждого куста $k_{i} ; a_{j_{k_{i}}}$ - вершины $k_{i}$ куста с внутренним индексом $j_{k_{i}}$. Выражение (3) уже явно демонстрирует цепной характер ассоциативности, причем каждое подмножество кустов инцидентно связывается с соответствующим подмножеством кустов более высокого уровня.

Примеры ДГК координационного типа даны на рис. 1б, 2б, 2в. По нашему мнению, эти ДГК можно считать сложными системами, а категорию сложности характеризовать этажностью ДГК, высотой дерева и развитостью кустов на иерархиях. Очевидно, чем больше кустистость на данной иерархии и чем больше высота ДГК, тем сложнее дерево в целом.

\section{4. ДГК В УСЛОВНОЙ ЛОГИКЕ, ЕГО СИМПЛИЦИАЛЬНАЯ ДЕКОМПОЗИЦИЯ. ДГК КАК ТРОЙКА ОБЪЕКТОВ}

Полученные графы (приведенные на рис. 1б, 2б, 2в) удобно записать в условной логике формирования. Как всякие древесные графы, они будут задаваться числом вершин и соответствующих связей. На данном этапе мы ведем рассмотрение в приближении исходящих кустов. Но “кустовую” запись еще надо получить. Самое простое - это записать ДГК в следующей форме:

$$
G=\left\{N=\sum_{i} \sum_{j_{i}} n_{j_{i}} ; \bigcup_{i}^{n_{i}} \bigcup_{j_{i}}^{n_{j_{i}}} x\left(l_{j_{i+1}} / l_{j_{i}}\right) ; k\left(l_{j_{i}}\right)\right\},
$$

где $i$ - уровень иерархии, $j_{i}$ - вершина на $i$-м уровне, $k\left(l_{j_{i}}\right)$ - степень ветвистости, $x\left(l_{j_{i+1}} / l_{j_{i}}\right)$ - исходящее ребро от $j_{i}$ до $j_{i+1}$ (выражение записано в терминах условной логики), $n_{j_{i}}$ - число вершин $j_{i}$ на $i$-м уровне, $N$ - общее число вершин ДГК, $\bigcup_{i} \bigcup_{j_{i}}-$ операторы объединения по вершинам $j_{i}$ и уровням $i$. В такой записи есть один существенный недостаток: хотя применяется условная логика разрастания ДГК, нет явного указания на кустовой характер самого разрастания. Последнее обстоятельство требует привлечения соответствующих декомпозиционных методов. В теории сложных систем [21] существует важный тип декомпозиции, который известен как симплициальная декомпозиция, характеризующийся весьма важным для нас свойством. Фактически это уже область системного анализа [22], в котором подразумевается разбиение суперДГК на простейшие элементы, подобные в некотором смысле исходному объекту. Нетрудно догадаться, что такими модулями будут являться исходящие кусты. Вполне очевидно, что парциальные кусты, по крайней 
мере в топологическом плане, подобны суперДГК. Всякий куст можно представить как сумму исходящих ветвей:

$$
g_{x}\left(K\left(l_{j_{i}}\right)\right) \equiv \sum_{j_{i+1}}^{K\left(l_{j_{i}}\right)} x\left(l_{j_{i+1}} / l_{j_{i}}\right) .
$$

В этом выражении рассматриваются парциальный куст ветвистости $K\left(l_{j_{i}}\right)$, стартующий из соответствующей вершины. Суммирование производится для парциального куста по индексу $j_{i}$, а $x\left(l_{j_{i+1}} / l_{j_{i}}\right)$ - ветви в условной логике.

С учетом (5) из общего задания ДГК (4) как раз и вытекает симплициальная кустовая декомпозиция:

$$
G=\left\{N ; \sum_{i=1}^{n_{i}} \sum_{j_{i}}^{n_{j_{i}}} g_{x}\left(K\left(l_{j_{i}}\right)\right)\right\} .
$$

Это выражение по сравнению с представлением (4) уже использует в явном виде кустовую симплициальную декомпозицию ДГК иерархического типа. Однако описание (6) также неполное. Напрашивается следующий шаг - введение некоторой меры $\mu\left(g_{l_{i}}(k)\right) \equiv \mu\left(g\left(k\left(l_{i}\right)\right)\right)$, в том числе и вероятностной, на кустах графа. Если это вероятностная мера, то она фактически определяет распределение кустов по ветвистости в тангенциальном направлении. В целом можно говорить об объединении мер на кустах $\bigcup_{l_{i}} g_{l_{i}}(k)$, которое представляет собой тангенциальную свертку кустов $l_{i}$ на уровне $i$. Тогда наш древесный граф, наделенный мерой или соответствующей вероятностной структурой, запишется следующим образом:

$$
G=\left\{N=\sum_{i} n_{i} ; \bigcup_{i} \bigcup_{l_{i}} g_{l_{i}}(k) ; \mu\left(g_{l_{i}}(k)\right)\right\}
$$

Таким образом, координационные ДГК задаются тройкой объектов: определяется множество связок вершина-ветвь как совокупность элементарных объектов, затем вводятся пространство вероятностных объектов или кустов и, наконец, мера на последних. Подразумевается, что в выражении (7) действует стандартная булева логика парциальных вероятностных событий кустового типа в представлении независимых исходящих кустов.

Для дальнейшего продвижения необходимо построить на ДГК некоторую сетевую процедуру в смысле де Посселя [23]. Предполагается, что будет осуществляться счетно-аддитивное разбиение на специальные кустовые подграфы, которое удовлетворяет условиям $\sigma$-алгебры. Последнее автоматически выполняется в приближении исходящих кустов. Конкретизуем систему $A_{i}$ подмножеств в духе де Посселя. Для этого выберем какую-либо ячейку нашей сетки и построим центральное дерево первой координации. Совокупность ветвей $\left\{x\left(l_{1} / k_{0}\right) ; \mu\left(l_{1} / k_{0}\right)\right\}$ с начальной вероятностной мерой образует множество первого ранга. На следующей координационной 
сфере реализуются кусты $\left\{x\left(l_{2} / k_{1}\right) ; \mu\left(l_{2} / k_{1}\right)\right\}$, где вероятностные меры, описывающие распределение кустов по степени ветвистости, уже могут быть и неравномерными. Далее применяется итеративная процедура. Кусты различного ранга в представлении исходящих кустов попарно не пересекаются, а система $\left\{A_{k_{i}}^{m_{i}}\right\}_{n}$ кустовых подмножеств доставляет объединение их во все супердерево с соответствующей иерархией вероятностных мер для кустов смежности. Таким образом реализованная ранговая система кустовых подграфов координаций позволяет интерпретировать ДГК как сеть в духе де Посселя.

В отличие от общепринятого похода [24], перейдем к энтропийным функционалам, которые определены на сети в смысле де Посселя для наших ДГК. Запишем дискретную счетно-аддитивную функцию энтропии $h\left(A_{i}\right)$ ранговых подмножеств:

$$
h\left(A_{i}\right)=\sum_{x_{k_{i}} \in A_{i}} f\left(x_{k_{i}} \in A_{i}\right) \Delta S\left(x_{k_{i}} \in A_{i}\right),
$$

где $x_{k_{i}}$ - смежности (ветви кустов с номером $k_{i}$ на $i$-й иерархии), $A_{i}-$ совокупность исходящих кустов $i$-й иерархии, $h\left(A_{i}\right)$ - энтропия вероятностных мер иерархии, $S\left(r_{(i+1) / i}\right)$ - ранговая геометрическая энтропия ДГК для $(i+1) / i$-й координационной сферы.

В нашем случае энтропийные "заряды" в (8) существенно неотрицательные (положительные), однако это не сужает общности рассмотрения. Система сфер (слоев координаций) образует сплошное покрытие: $\left\{A_{1}\right\} \prec\left\{A_{2}\right\} \prec \cdots \prec\left\{A_{m}\right\}$, где $\prec-$ объемлющий символ упорядоченности уровней древесной иерархии вплоть до $m$-го ранга. Каждому уровню сопоставляется два ряда энтропий: $h\left(A_{1}\right), h\left(A_{2}\right), \ldots, h\left(A_{m}\right) \geqslant 0$ и, соответственно, геометрическая (ранговая) энтропия $S(1), S(2), \ldots, S(m)$, где $S(r)=$ $\lg r \leqslant \lg m, r=1,2, \ldots, m, m \rightarrow \infty$. Энтропия $S(\cdot)$ - монотонная выпуклая функция от ранга (уровня) ДГК, $S(r) \rightarrow \infty$ при $r \rightarrow \infty$. Заметим, что ряд вероятностных энтропий не является упорядоченным, в отличие от геометрической энтропии. За производящую функцию координационных сфер естественно принять ступенчатую ранговую зависимость, и тогда интегральная энтропийная мера Радона запишется как

$$
\begin{aligned}
F(m) & =h\left(\bigcup_{1}^{m} A_{i}\right)=\sum_{x_{k_{i}} \in A_{1}}^{x_{k_{i}} \in A_{m}} f\left(x_{k_{i}} \in \bigcup_{1}^{m} A_{i}\right) \Delta S((i+1) / i)=\sum_{A_{1}}^{A_{m}} h\left(A_{i}\right)= \\
& =\sum \kappa\left(A_{i}\right) f\left(A_{i}\right) \Delta S\left(A_{i}\right) \Rightarrow \sum_{i=1}^{m} f\left(S_{i}\right) \Delta S_{i}=H\left(S_{n}\right),
\end{aligned}
$$

где $\kappa\left(A_{i}\right)$ - (четкие) индикаторы системы сфер де Посселя $\left\{A_{i}\right\}, f\left(A_{i}\right)$ - производная Радона-Никодима вероятностной энтропии по геометрической энтропии. Тогда в соответствии с (9) плотность дискретной функции координационных сфер имеет вид

$$
f\left(S_{m}\right)=\frac{\Delta F(m)}{\Delta S[m]},
$$


что представляет собой дискретную версиию производной Радона-Никодима $d H\left(S_{m}\right) / d S_{m}$ по схеме де Посселя на ДГК (очевидно, что при вычислении производной Радона-Никодима предполагается инфинитезимальный подход с соответствующим предельным переходом, что, конечно, невозможно в дискретных цепях). Для нас важен тот факт, что, оказывается, производная Радона-Никодима вероятностной энтропии по геометрической энтропии ДГК (10) на сети де Посселя равна фрактальной размерности ДГК.

\section{5. СВОЙСТВА КООРДИНАЦИОННЫХ ДГК}

Укажем наиболее важные, по нашему мнению, математические свойства отображения сеток, решеток в координационные ДГК.

1. Из рис. 1б, 2б, 2в видно, что наши ДГК наследуют полярную геометрию в плоскости укладки $\mathbb{R}_{2}$. Поэтому естественно выделить радиальное и тангенциальное направления на ДГК. Как видно из предыдущего рассмотрения, по индексу $i$ в радиальном направлении ДГК выполняется строгая эстафетность. Последнее свойство является следствием вышеприведенной цепочной логики построения ДГК по сеточным, решеточным структурам. Несколько забегая вперед, мы назвали это свойство марковским развертыванием, поскольку каждый уровень зависит только от единственного предыдущего уровня. Стоит заметить, что данное замечание относится именно к исходящим кустам.

Если говорить о тангенциальном представлении ДГК, то наиболее заметным является факт локальной внутриуровневой межкустовой пересекаемости, $\cdots \bigcap g_{k_{i}-1} \bigcap$ $g_{k_{i}} \cap g_{k_{i}+1} \bigcap \cdots \neq 0$, где $g_{k_{i}}, g_{k_{i} \pm 1}$ - смежные кустовые подграфы, но это свойство выполняется в приближении входящих кустов. По этой причине рассматриваемые нами ДГК совсем не являются деревьями Бете, даже если разрешить для последних случайную ветвистость. Деревья Бете - это топологически "бедные" деревья. Они принадлежат графам типа "леса" и не являются координационными ДГК. В литературе [19], [20] распространены деревья Бете-Кэли, но они рассматриваются в методологии графов типа “леса". Для наших координационных графов налицо межкустовая локальная пересекаемость, но марковское свойство при этом не нарушается. В свое время мы ввели термин "марковские джунгли", которые гораздо сложнее, избыточнее по сравнению с обобщенными деревьями Бете-Кэли [11], [12].

2. "Богатство" ДГК надо понимать в том смысле, что наряду с прямым потоком существует и обратный поток, который принципиально отсутствует в древесных графах Бете-Кэли. Именно локальная пересекаемость в приближении входящих кустов дает возможность рассматривать два встречных потока на ДГК, и тогда можно говорить о степени необратимости перколяционных потоков в случае их неравенства. Упомянутые перколяционные потоки идут от центра к периферии и обратно, что устанавливает на ДГК некоторую симметрию структуры отношений. В качестве таких отношений в графодинамике [25] используются термины подчинения-командования, которые перколируют именно в логическом плане. Однако типовые функции подчинения для графов типа “леса", известные в графодинамике [25], для "марковских джунглей" не проходят. Аппарат таких функций 
был расширен до дифференциальной формы функций подчинения-командования, которые построены из $\delta$-функций Дирака с амплитудами [11], [12].

3. В рамках теории сложных систем [21], как мы уже упоминали, наиболее важной для нас является методика симплициальной декомпозиции ДГК. Каждый куст рассматривается как парциальный ультрасимплекс, а сама декомпозиция ДГК базируется на понятии симплициального ультраметрического комплекса. ДГК в целом является суперультрасимплексом. Тогда удобно записать кустовую схему ДГК в форме

$$
G=\bigcup_{i}^{n} \bigcup_{K_{i}}^{m}\left\{l_{\rho}\left(K_{j_{i}}\right)\right\}=\sum_{K, i}^{m, n} U_{l_{K, i}^{\text {simp }}}, \quad U_{l^{\text {simp }}}=\left\{l_{\rho}\left(K_{j_{i}}\right)\right\},
$$

где $i$ - радиальный индекс (номер уровня иерархии) ДГК, $K$ - тангенциальный индекс куста, $j$ - внутрикустовой индекс ветви, $\left\{l_{\rho}\left(K_{j_{i}}\right)\right\}-\rho$-я ветвь $j$-го куста $K$-й ветвистости, $U_{l_{\text {simp }}}$ - ультрасимплекс, представляющий соответствующий парциальный куст. Наличие симплициальной декомпозиции ДГК (11) подсказывает некоторый принцип структурного топологического подобия, которое находит совершенно разную трактовку в двух разделах современной математической науки. Один из них - это фрактальная геометрия, а другой принадлежит теории сложных систем или, более узко, теории симплициальных комплексов [21], [26]. Пожалуй, впервые аппарат ДГК получил удобное применение к процессу фрактализации в работе [27]. Каждый ультрасимплекс (11) в топологическом плане подобен суперультрасимплексу. Свойство подобия является необходимым условием фрактальности [28]. Поэтому можно указать на весьма жесткую корреляцию свойства симплициального подобия и фрактальности ДГК. Это условие не является достаточным, оно всего лишь необходимо при оценке фрактальных размерностей, но оно позволяет, рассматривая ДГК на рис. 1б, 2б, 2в, автоматически мыслить в терминах фрактальности.

4. Отношения инциденций в классическом плане характеризуют прямое соседство, смежность (прямой родственник). ДГК на приведенных выше рисунках показан в виде укладки на евклидову плоскость $\mathbb{R}_{2}$. Сразу видно, что внутренняя геометрия ДГК не является евклидовой и не согласована с внутренней геометрией плоскости укладки. В результате на рис. 1б, 2б, 2в все связи (ветви) вовсе не выглядят одинаковыми, хотя все они единичные. Очевидно, что на ультрасимплексах можно задать ультраметрику. Тем самым внутренняя геометрия ДГК является ультраметрической, и при укладке на $\mathbb{R}_{2}$ претерпевает искажения. Однако все ветви, ребра надо считать строго единичными.

5. Стоит обратить особое внимание на тот факт, что в приближении входящих ветвей, как мы упоминали выше, на всех уровнях присутствует локальная межкустовая пересекаемость соседних кустов, но при сохранении свойства эстафетности (марковости) в радиальном направлении. Алгебраическое свойство пересекаемости дает возможность сформулировать понятие связности на ДГК в приближении входящих кустов. Такого понятия в классических графах Бете-Кэли [19], [20] нет в принципе. Именно по этой причине на них отсутствует обратный поток перколяции. Наличие тангенциальной локальной связности позволяет построить некоторые 


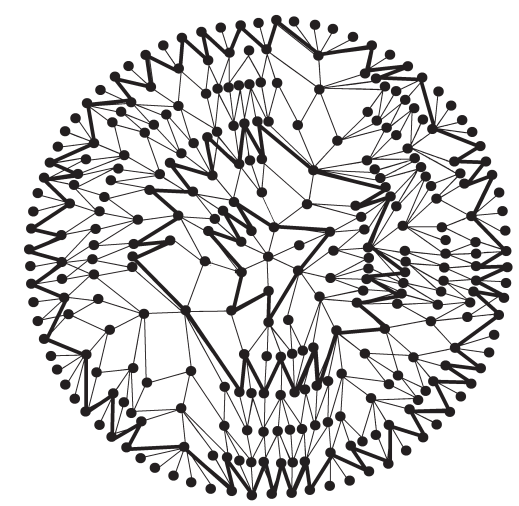

Рис. 3. Скорлупы Мандельброта на ДГК для кварцевого стекла КУВИ-1.

замкнутые фронты, которые обладают “винеровской” топологией и являются геодезическими ломаными линиями на каждом уровне иерархии. Это скорлупы Мандельброта (рис. 3). Скорлупа Мандельброта является фрактальным обобщением ("жирным" фракталом в терминах работы [29]) гладких выпуклых фронтов Гюйгенса. Концентрически вложенные скорлупы являются представлением волновой фрактальной перколяции на ДГК.

Учет ультраметрики ДГК позволяет ввести на скорлупе Мандельброта естественную меру: $L_{i}=\sum_{k_{i}=0}^{m_{i}} l_{k_{i}}$, где $l_{k_{i}}-$ единичная длина ветви геодезического ультрасимплекса. Рассматривая длину $C_{i}=2 \pi i$ гипотетической окружности на каждом уровне иерархии ДГК, введем показатель "жирности" скорлупы Мандельброта $\xi_{i}=L_{i} / 2 \pi i$. После несложных преобразований получаем оценку фрактальности скорлупы Мандельброта на $i$-м уровне ДГК через показатель ее “жирности":

$$
d_{\mathrm{MSh}}\left(\xi_{i}\right)=1+\frac{\ln \xi_{i}}{\ln (i+1)},
$$

где $i=1,2 \ldots-$ номера уровней иерархии ДГК, $\ln (i+1)$ - ультраметрическая высота ДГК на $i$-м уровне. Для получения общей оценки фрактальности всего ансамбля скорлуп Мандельброта (см. рис. 3) можно использовать усреднение показателя (12) по индексу $i$ или остановиться на асимптотической характеристике.

6. Перколяцию можно рассматривать и в дуальном аспекте. Полезно вспомнить классическое понятие лучей, ортогональных к волновым гладким выпуклым фронтам Гюйгенса. В нашем случае дуальными объектами к скорлупам Мандельброта являются стримеры [2]. Мы назвали их в наших ранних работах [15], [30] "лохматыми" фракталами (см. рис. 4, 5).

Переход к стримерному представлению - это переход к новому типу симплициальной декомпозиции суперДГК. Теперь в качестве подсистемы выбирается ансамбль стримеров, который, в свою очередь, должен удовлетворять условию полноты и обладать свойствами $\sigma$-алгебры (рис. 5); усреднение парциальных фрактальных характеристик по этому ансамблю и даст оценку стримерной фрактальности ДГК.

4 Теоретическая и математическая физика, т. 164, № 1, 2010 г. 


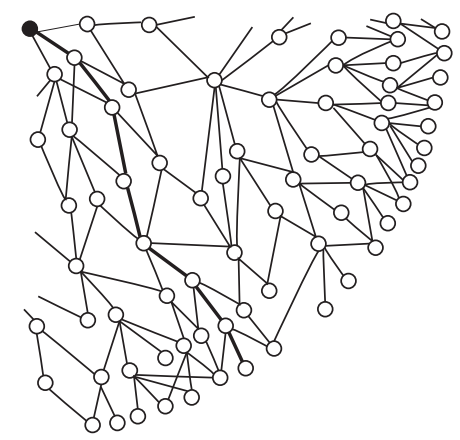

a

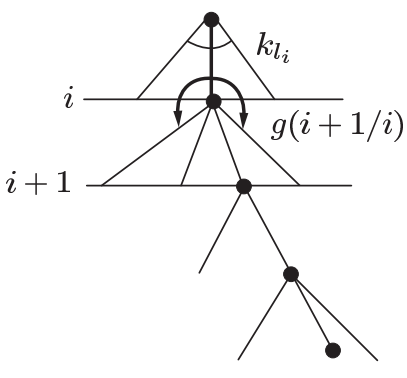

б

Рис. 4. Схема построения стримера на ДГК (а) и логика его формирования (б).

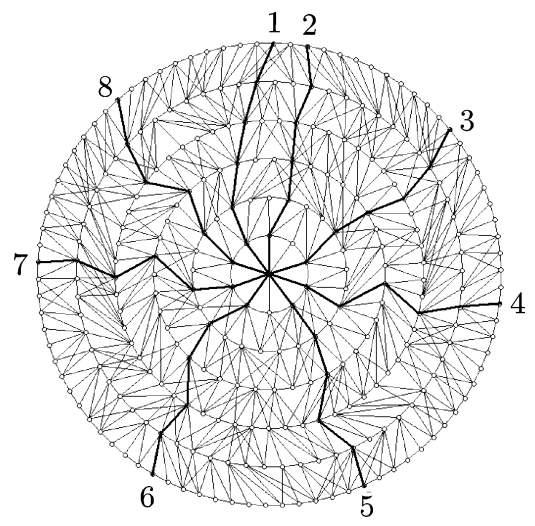

Рис. 5. $\sigma$-Ансамбль стримеров на ДГК Пенроуза.

В стримерном представлении ДГК мы должны пользоваться выражением (7) и фактически задать соответствующую тройку объектов. Поэтому необходимо сначала построить логику формирования стримера, затем на последовательностях кустов каждый раз будет реализован стохастический выбор конкретных ветвей. При этом предполагается, что стохастический оператор выбора задается соответствующей мерой, которая определяется степенью ветвистости парциальных кустов в их цепочке. Конкретная итерация процедуры выбора ветвей - это и есть стример. Своим происхождением он обязан итеративной последовательности стохастических операторов выбора.

Схематически стример представлен на рис. 4, 5. Как мы видим, всякий стример является цепью логических конструкций, а также вероятностных событий на них. Отметим, что итерации выбора реализуются в усеченных алгоритмах. Фактически каждый шаг алгоритма выбора сводится к некоторому условному выбору следую- 
щего типа: случайно выбирается ветвь из некоторого $l_{i}$-го куста на $i$-м уровне, что автоматически влечет за собой выбор куста на $(i+1)$-м уровне.

Таким образом, простейшим вероятностным событием является событие $V_{l_{i}} \in g\left(k_{l_{i}} \wedge g_{l_{i+1}}\right)$, где $V_{l_{i}}$ означает ветвь конкретного $l_{i}$-го куста на $i$-м уровне; она принадлежит соответствующему дереву, аргумент которого определяется ветвистостью $k_{l_{i}}$; символ $\wedge$ означает логику объединения конкретной ветви предыдущего куста и последующего "немого" куста $(i+1)$-го уровня (см. рис. 4б). Логика стримера запишется в следующем виде:

$$
\begin{aligned}
\widehat{S}_{10} R & \Rightarrow\left\{V_{l^{\prime}}(1 / 0) \wedge g_{l^{\prime \prime}}(2 / 1)\right\}, \\
\widehat{S}_{21}\left(k_{l^{\prime}}(1)\right) & \Rightarrow\left\{V_{l^{\prime \prime}}(2 / 1) \wedge g_{l^{\prime \prime \prime}}(3 / 2)\right\}, \\
\widehat{S}_{32}\left(k_{l^{\prime \prime}}(2)\right) & \Rightarrow\left\{V_{l^{\prime \prime \prime}}(3 / 2) \wedge g_{l^{I V}}(4 / 3)\right\},
\end{aligned}
$$

где явно указан наш составной элемент выбора, состоящий из ветки $V_{l^{\prime \prime}}\left((2 / 1) ; k_{1}\right)$ конкретного куста $V_{l^{\prime \prime}}(2 / 1)$ верхнего уровня и "немого" куста $g_{l^{\prime \prime \prime}}(3 / 2)$ нижнего уровня. Операторы стохастического выбора (13) действуют на соответствующих кустах. Количество неопределенности, которое преодолевают данные операторы, зависит лишь от ветвистостей $k_{0}, k_{1}, k_{2}, \ldots$ этих кустов. Цепь операторов (13) можно рассматривать как независимую. Следовательно, количество неопределенности на всем стримере должно подчиняться условию аддитивности.

Количество неопределенности удобно охарактеризовать энтропией цепочки кустов:

$$
\begin{aligned}
& H(R) \equiv H\left(g_{l^{\prime}}(1 / 0) ; k_{0}\right)=\lg k_{0}, \\
& H\left(g_{l^{\prime \prime}}(2 / 1) ; k_{1}\right)=\lg k_{1}, \\
& H\left(g_{l^{i+1}}((i+1) / i) ; k_{i}\right)=\lg k_{i} .
\end{aligned}
$$

Если теперь перейти к стримеру $m$-й длины, то с точки зрения теории вероятностей стример будет задаваться $m$-м пересечением кустов, и в соответствии с (14) суммарная энтропия стримера запишется как

$$
H_{m}\left(g_{l^{\prime}} \wedge g_{l^{\prime \prime}} \wedge g_{l^{\prime \prime \prime}} \wedge \cdots\right)=\sum_{i} \lg k_{l_{i}}=\lg \prod_{i} k_{l_{i}}
$$

В данной формуле предполагается, что введены абсолютные вероятностные меры ветвистости соответствующих кустов. Удобно получить для выражения (15) оценку сверху, используя одно из простейших неравенств для выпуклых функций:

$$
H_{m}\left(g_{l^{\prime}} \wedge g_{l^{\prime \prime}} \wedge g_{l^{\prime \prime \prime}} \wedge \cdots\right)=\sum_{i} \lg k_{l_{i}} \leqslant \sum_{i}\left(k_{l_{i}}-1\right) \leqslant \sum_{i} k_{l_{i}} .
$$

Эта оценка позволяет определить суммарное значение неопределенности в виде простой суммы ветвистостей соответствующих кустов, по которым прошел стример (рис. 4, 5). 
Можно дать геометрическую иллюстрацию в ортогональных пространствах с метрикой Хемминга, но при переменной размерности, которая соответствует длине стримера на ДГК. Оператор стохастического выбора действует на соответствующих кустах, которые подчиняются цепной логике. Мощность оператора выбора определяется ветвистостью соответствующего куста. Именно значениям ветвистостей $k_{l_{i}}$ отводится роль проекций на ортогональном репере $O\left(k_{0}, k_{l_{1}}, \ldots, k_{l_{m}}\right)$ (рис. 4). Вообще говоря, в дальнейшем подразумевается репер переменной размерности по $m$, причем целочисленный. Метрика в духе Хемминга такого пространства будет простой суммой ветвистостей. Однако согласно предыдущим рассуждениям этот результат является линейной мажорантой логарифма (16). Более последовательное задание пространства выбора, таким образом, следует проводить на логарифмическом репере $O\left(\lg k_{0}, \lg k_{l_{1}}, \ldots, \lg k_{l_{m}}\right)$. Вектор (точка в этом пространстве) характеризуется длиной $\sum_{i=1}^{m} \lg k_{l_{i}}$, равной суммарной энтропии пространства неопределенности.

ДГК в целом можно представить как $\sigma$-ансамбль стримеров, мощность которого оценивается стартовым координационным числом. В общем случае число таких полностью развязанных стримеров (рис. 5) будет иметь начальное координационное число как верхнюю оценку. Приведем схему оценки стримерной фрактальности для ДГК в целом.

На стримере $m$-й высоты мы строим дивизор $D_{l_{\mathrm{str}}}(m)=\prod_{k_{1}}^{k_{m}} k_{l}$. Затем находим парциальную энтропию дивизора $l$-го стримера $m$-й высоты: $h\left(D_{l_{\mathrm{str}}}(m)\right)=$ $\ln D_{l_{\mathrm{str}}}(m)$. Параллельно формируется геометрическая энтропия $S(m)=\ln m$ ДГК. Парциальную энтропию ветвистости стримера можно рассматривать как некоторую функцию $h_{D}[S(m)]$ в смысле Радона от геометрической энтропии, где обе энтропии принадлежат одному классу, и их можно объявить неотрицательными мерами Радона. Теперь можно образовать производную Радона-Никодима, линеаризация которой доставит фрактальную размерность стримера:

$$
d_{l_{\mathrm{str}}}(m)=\frac{\ln \ln D_{l_{\mathrm{str}}}(m)}{\ln m}
$$

Ансамбль стримеров наделен $\sigma$-свойством, поэтому фрактальную размерность ДГК в стримерном представлении можно получить простым усреднением парциальных фрактальных размерностей (17) стримеров.

7. Исходя из дуального представления общего потока на ДГК можно говорить об обобщенной перколяции, в которой участвуют не только скорлупа Мандельброта (рис. 3), но и стримеры (рис. 4, 5). Общий поток на ДГК может быть сформулирован в виде прямой суммы этих компонент. При этом фрактальные размерности скорлуп Мандельброта (12) и стримеров (17) будут просто складываться. В этом случае размерность обобщенного потока, как правило, будет превосходить топологическую размерность среды, что мы называем феноменом сверхразмерной перколяции [30]. Однако эта "сверхразмерность" будет всегда меньше топологической размерности минимального гладкого пространства, которое и является пространством вложения. 


\section{6. ПЕРКОЛЯЦИЯ ЭНТРОПИЙНОГО ФУНКЦИОНАЛА НА КВАРТЕТНОМ АЛФАВИТЕ МОЗАИКИ ПЕНРОУЗА}

Пентасимметричная мозаика Пенроуза представляет собой один из фундаментальных квазикристаллических объектов и уже поэтому нуждается в специальном исследовании. Как видно из рис. 2б, 2в, ДГК Пенроуза содержит два типа вершин и два типа связей. Вершинная информация базируется только на дуальном алфавите "золотых" ромбов ( $g$-ромбов), которые получены объединением двух треугольников Робинсона по “золотой” стороне. Назовем это $q$-компонентой алфавита. Далее, $p$-компонентой назовем координационные связи, ветви, соответствующие реберной и точечной координациям. Тем самым мозаику Пенроуза и ее ДГК разумно задать на квартетном алфавите $[2 q \times 2 p]$.

Можно построить матрицу "ромб-тип координаций", матричными элементами которой являются вероятности (частоты) появления этих четырех символов [11], [13], [31]. Данная матрица показана в следующей таблице.

\begin{tabular}{|c|c|c|c|}
\hline & $\nu$ & $c$ & $P\left(\omega_{1} ; \omega_{2} / \nu \vee c\right)$ \\
\hline$\omega_{1}$ & 0.427 & 0.191 & $P\left(\omega_{1} / \nu \vee c\right)=P_{0}\left(\omega_{1}\right)=0.618$ \\
\hline$\omega_{2}$ & 0.264 & 0.118 & $P\left(\omega_{2} / \nu \vee c\right)=P_{0}\left(\omega_{2}\right)=0.382$ \\
\hline
\end{tabular}

В таблице приняты следующие обозначения: $\omega_{1}$ - "тупой" ромб, $\omega_{2}$ - "острый" ромб, $\nu$ - вершинный (точечный) контакт, $c$ - реберный контакт. Ранжированная статистика матрицы вероятностей приведена на рис. 6а. Благодаря этой матрице сразу видна вероятностная роль “золотистости”. Чисто геометрическое свойство нашло вероятностную интерпретацию, что далеко не очевидно. Частота встречи "тупого" $g$-ромба при любом виде контакта, $P\left(\omega_{1} / \nu \vee c\right)=P_{0}\left(\omega_{1}\right)$, как раз равна "золотому" числу, а ее дополнение до единицы равно вероятности встретить "острый” $g$-ромб независимо от типа контакта: $P\left(\omega_{2} / \nu \vee c\right)=P_{0}\left(\omega_{2}\right)$.

Квартетный алфавит естественно ранжировать и построить ранжированную статистику для начального и асимптотического векторов в марковском представлении [31], что и показано на рис. 6а. Из самой матрицы и рис. 6а видно, что реберные контакты у обоих типов $g$-ромбов самые “дорогие”, а потому маловероятные. Точечные контакты более "дешевые" и образуют на рис. 6а левый отрезок более высоких значений вероятностей.

В логарифмическом масштабе хорошо видно, что ранжированная статистика является степенной с показателем $\gamma \leqslant 1$. Это означает, что она принадлежит к классу Ципфа-Парето-Мандельброта. Из этого следует, что мозаику Пенроуза можно трактовать как текст некоторого языка с установленной выше грамматикой [31].

Изложенный выше подход можно развить, рассмотрев двухуровневое бинарное дерево (рис. 7). Первый уровень дерева указывает на число ромбических разбиений, вероятностные свойства которых обсуждались выше. На втором уровне к ромбам добавляются два вида контактов, точечный и реберный, что дает алфавит $[2 q \times 2 p]$. 


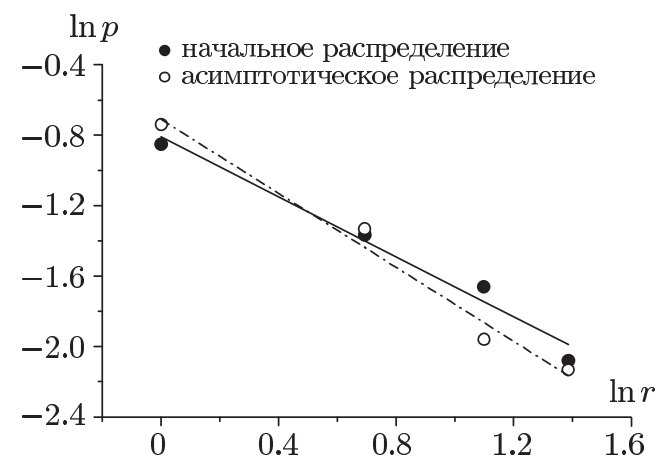

a

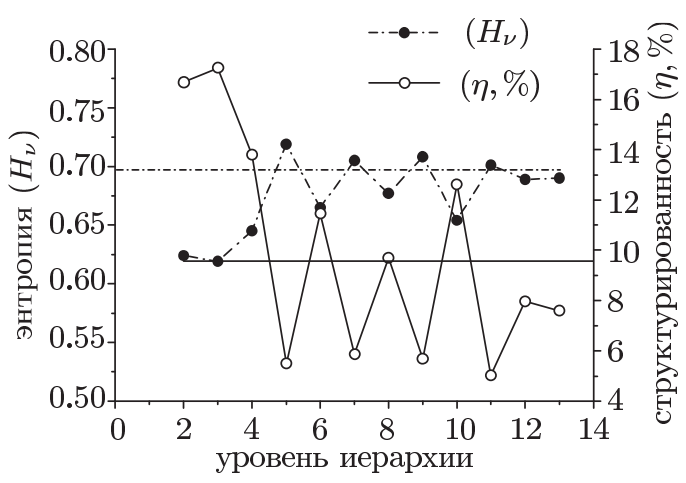

б

Рис. 6. Вероятностная структура алфавита ДГК Пенроуза (а); перколяция энтропийного функционала $H_{\nu}$ и коэффициент структурированности $\eta$ для мозаики Пенроуза (б).

На рис. 7 показано двухуровневое бинарное дерево, на котором первый уровень иерархии соответствует дуальному алфавиту, а второй уровень - квартетному. Там же приведены внутренние отношения на дуальном и квартетном уровнях соответствующих вероятностей. Все они управляются "золотыми” числами. Аналогичные отношения на квартетном уровне (см. рис. 7) управляются суммой “золотых" чисел $\alpha_{1}+\alpha_{2}$. Кроме того, можно ввести более сложное двойное отношение между бинарным и квартетным уровнями:

$$
\frac{\omega_{1} / \omega_{1}^{\nu}}{\omega_{1} / \omega_{1}^{c}}=\frac{\omega_{2} / \omega_{2}^{\nu}}{\omega_{2} / \omega_{2}^{c}}=\frac{1}{\alpha_{1}+\alpha_{2}} .
$$

Таким образом, оба уровня алфавита Пенроуза пронизаны “золотой” симметрией, что видно из таблицы, рис. 7 и выражения (18). При этом квартетный алфавит подчиняется статистике Ципфа-Парето-Мандельброта. Из этого следует, что квартетный алфавит $[2 q \times 2 p]$ имеет определенные лингвистические функции.

Приведенное беглое рассмотрение позволяет высказать гипотезу, что мозаика Пенроуза может быть интерпретирована как двумерный текст [31] на трехуровневом дуальном алфавите [32] с древесно-графовой грамматикой. Завершим этот раздел рассмотрением ключевого момента, который связан с энтропийной перколяцией на ДГК Пенроуза. Мы считаем, что характер энтропийной перколяции на древесных графах может быть мерой порядка-беспорядка. На рис. $6 б$ приведен итоговый результат энтропийной перколяции на квартетном уровне алфавита $[2 q \times 2 p]$. Четко видна ярко выраженная периодичность. Энтропия квартетного алфавита, равная $0.697 \ldots$, которая является его емкостью, в точности совпадает со средней перколяционной энтропией (см. рис. 6б) квартетного алфавита. Последнее обстоятельство означает, что на каждом уровне перколяции система Пенроуза использует ту же самую вероятностную структуру алфавита $[2 q \times 2 p]$ с максимально возможным 


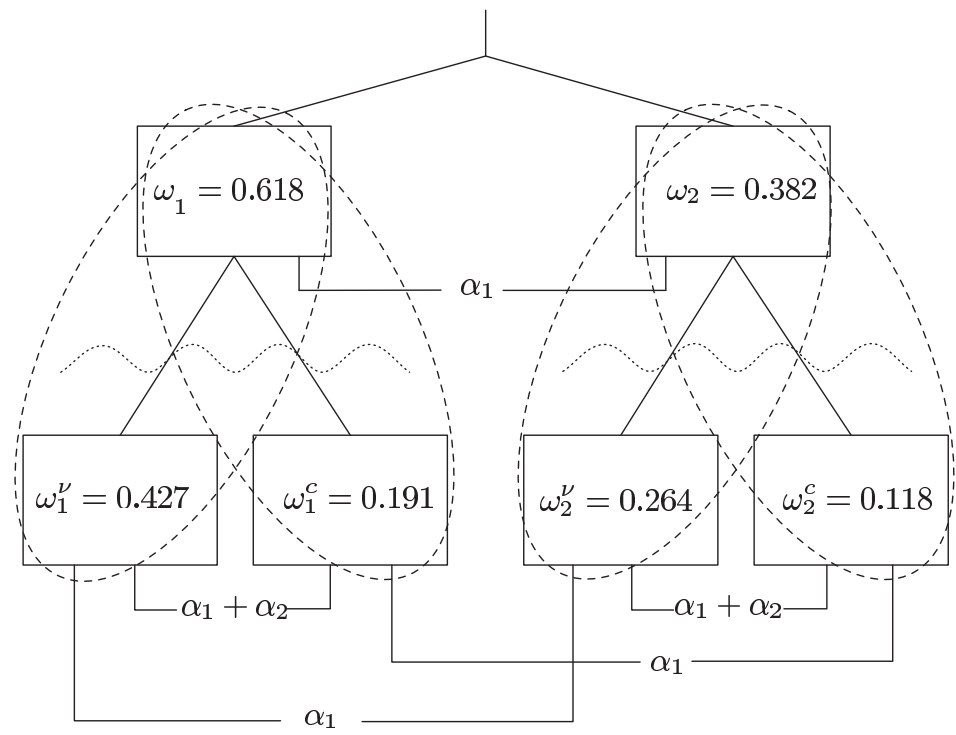

Рис. 7. Двухуровневое бинарное дерево алфавита $[2 q \times 2 p]$.

квартетным разнообразием. Кроме того, на рис. $6 б$ показан коэффициент структурированности, который может быть также использован для оценки степени хаотичности. Отсюда можно заключить, что ДГК Пенроуза, а с ним и сам паркет характеризуются уровнем хаотичности порядка $90 \%$.

\section{7. ПЕРКОЛЯЦИЯ ИНФОРМОДИНАМИЧЕСКИХ ФУНКЦИОНАЛОВ НА ДРЕВЕСНЫХ ГРАФАХ КЭЛИ}

Введенные нами ДГК, отображающие сеточные и решеточные структуры, допускают симплициальную декомпозицию. А это, в свою очередь, позволяет использовать теорию перечисления древесных графов [26]. Для каждого уровня ДГК строится перечисляющий полином (ПП), каждый член которого представляет куст соответствующей ветвистости с коэффициентами, равными числу кустов данной ветвистости на иерархии:

$$
\Pi_{i}\left(x^{r}\right)=\sum_{k=0}^{r} T_{i}\left(x^{k}\right) x^{k},
$$

где $x^{k}$ - куст $k$-й ветвистости; $T_{i}\left(x^{k}\right)$ - коэффициент, отвечающий за число кустов $k$-й ветвистости на $i$-м уровне ДК, $r$ - ранг ПП. Можно эквивалентным образом сопоставить ПП распределения. Вводя нормировку на единицу (деля на $N_{i}=\sum_{k=1}^{r} T_{i}\left(x^{k}\right)$ ), получим вероятностный ПП (ВПП)

$$
\Pi_{i}\left(x^{r}\right)=\sum_{k=0}^{r} t_{i}\left(x^{k}\right) x^{k},
$$


где $t_{i}\left(x^{k}\right)=T_{i}\left(x^{k}\right) / N_{i}$. Первый уровень перколяционной задачи можно понимать в смысле протекания по ДГК перечисляющих структур (19), (20). Характер симметрии ячеистых структур должен найти отражение как в поведении коэффициентов ПП, так и в степенях кустистости. Вообще говоря, полученные ВПП уже не стоит считать полиномами. Это, скорее, распределения кустов по ветвистостям. При необходимости можно допустить процедуру перенормировки.

Первый уровень перколяционной задачи относится к протеканию ПП или ВПП, т.е. к протеканию перечисляющих структур по ДГК. На этом уровне можно получить весь многообразный статистический анализ перколяции ВПП, но нас это не будет интересовать. По нашему мнению, следующий уровень перколяционной задачи на ДГК должен базироваться на некоторых функционалах от перечисляющих структур. Есть смысл особо выделить информодинамические функционалы [15], в частности энтропийные и дивергентные функционалы [24]. Последние могут выступать как соответствующие информационные метрики в перколяционной задаче на ДГК. Как всякая энтропия, введенные нами энтропийные функционалы должны удовлетворять необходимым и достаточным условиям теоремы Фаддеева-Фейнстейна. Запишем соответствующий энтропийный функционал в форме Вайда для наших ВПП на ДГК:

$$
H_{\mathrm{V}}\left[\Pi_{i}\left(x^{r}\right)\right]=\sum_{k=1}^{r} t_{i}\left(x^{k}\right)\left[1-t_{i}\left(x^{k}\right)\right]=\sum_{k=1}^{r} t_{i}\left(x^{k}\right) \overline{t_{i}\left(x^{k}\right)}
$$

где $t_{i}\left(x^{k}\right)$ - коэффициент ВПП, а $\left[1-t_{i}\left(x^{k}\right)\right]=\overline{t_{i}\left(x^{k}\right)}-$ его дополнение. $\mathrm{B}$ выражении (21) интересна логика образования энтропии Вайда. Она отличается от шенноновской и фактически является ее мажорантой.

Более нетривиальным функционалом от ВПП следует признать информационную дивергенцию Бонгарда [24]. Логика ее построения более сложна, чем дивергенции Кульбака [24]. Дивергенция Бонгарда является информационным расстоянием [33], и в форме Вайда она запишется следующим образом:

$$
\begin{aligned}
\operatorname{Biv}\left[t_{i}\left(x^{k}\right) ; t_{i+1}\left(x^{k}\right)\right] & \equiv B\left[t_{i}\left(x^{k}\right) ; t_{i+1}\left(x^{k}\right)\right]= \\
& =\frac{1}{2} \sum_{k=1}^{r}\left\{t_{i}\left(x^{k}\right)\left[1-t_{i+1}\left(x^{k}\right)\right]+t_{i+1}\left(x^{k}\right)\left[1-t_{i}\left(x^{k}\right)\right]\right\} .
\end{aligned}
$$

Данное выражение в логике Вайда соответствует расстоянию между парами смежных уровней ДГК, в то время как выражение (21) соответствует собственной энтропийной мере ВПП на $i$-м уровне иерархии ДГК.

Сделаем некоторые замечания по типу оператора перколяции. Изначально он действует на перечисляющих структурах, но его действие может быть определено и на функционалах от перечисляющих структур. Перечисляющие полиномы, вообще говоря, имеют различные нормы для различных уровней иерархий ДГК. ВПП, конечно, нормированы на единицу, но нормировочные константы каждый раз будут разными. Если бы в задаче перколяции сохранялась норма ВПП, то перколяционный оператор сводился бы к простому стохастическому оператору. Пусть $\widehat{S}-$ 
стохастический оператор, который действует на пространстве коэффициентов ВПП по правилу

$$
\widehat{S} \frac{T_{i}}{N_{i}}=\frac{T_{i+1}^{\prime}}{N_{i+1}}, \quad N_{i} \neq N_{i+1}, \quad T, T^{\prime} \in R_{T},
$$

где $T_{i}, T_{i+1}^{\prime}-$ коэффициенты ПП (19) в абсолютных единицах для $i$-го и $(i+1)$-го уровней иерархии, $R_{T}$ - пространство распределений (множество всех наборов неотрицательных коэффициентов, сумма которых равна единице), $N_{i}, N_{i+1}$ - нормировочные константы вышеуказанных распределений. Из выражения (23) нетрудно получить следующее:

$$
\operatorname{Amp}\left(N_{i} ; N_{i+1}\right) \widehat{S}=\widehat{S}_{\mathrm{Amp}}, \quad \operatorname{Amp}\left(N_{i} ; N_{i+1}\right)=\frac{N_{i+1}}{N_{i}} \Rightarrow \lambda_{\mathrm{Amp}} \widehat{S}=\widehat{S}_{\lambda},
$$

где $\widehat{S}_{\lambda}-$ квазистохастический оператор перколяции на ДГК. Введенный оператор перколяции базируется на обычном стохастическом операторе, но при этом в последних соотношениях имеется амплитудный множитель $\lambda_{\mathrm{Amp}}$, отличный от единицы. Последнее означает, что стохастический оператор подвергается растяжению или сжатию в соответствии с нормой распределений. Рассмотрение перколяционной задачи в энтропийных и дивергентных терминах составляет информодинамическую часть предлагаемой нами теории [11], [13], [15], [30], [32], [34]. По нашему мнению, именно перколяционные зависимости этих информодинамических функционалов и, соответственно, их критические индексы помогут получить общий метод решения задачи идентификации типа упорядочения сеточных структур и решеток.

\section{8. ЗАКЛЮЧЕНИЕ}

Предложен универсальный информодинамический метод диагностики характера порядка-беспорядка для решеточных, сеточных систем с достаточно общей топологией. Приведены логические аспекты отображения решеточных, сеточных структур в координационные ДГК. Они являются далеко идущим обобщением классических графов Бете, а также графов типа “леса". ДГК являются марковскими "джунглями". С точки зрения системного анализа сеточных и решеточных структур введена симплициальная кустовая декомпозиция на ДГК. Каждый парциальный куст есть элементарный ультрасимплекс, а ДГК в целом является суперультрасимплексом, допускающим разложение на элементарные модули, подобные дереву в целом. Отмечена корреляция симплициальности и фрактальности ДГК. Эта корреляция может быть использована как необходимое условие фрактальности сеточных и решеточных систем. В представлении входящих кустов установлен эффект локальной внутриуровневой межкустовой пересекаемости, который приводит к существованию скорлупы Мандельброта. Последняя является геодезической ломаной линией с "винеровской" топологией. Скорлупу Мандельброта можно рассматривать как фрактальное обобщение гладких фронтов Гюйгенса. Дуальным представлением к ней служат стримеры, которые являются обобщением простых лучей. Рассмотрена совместная перколяция во фронтально-лучевом представлении перечисляющих 
структур на ДГК и энтропийных функционалов от них. Именно энтропийная перколяционная зависимость и служит той универсальной мерой, благодаря которой можно диагностировать характер дальнего упорядочения решеточных и сеточных систем. Мозаика Пенроуза представляет собой двумерный текст на трехуровневом дуальном алфавите с древесно-графовой грамматикой. Перколяция энтропийного функционала на квартетном алфавите $[2 q \times 2 p]$ имеет осцилляторно-волновой характер, что является имманентным свойством пентасимметричной мозаики Пенроуза. Мы надеемся, что предлагаемый нами информодинамический метод может служить универсальным количественным идентификатором характера порядка-беспорядка любых решеточных и сеточных систем.

\section{Список литературы}

[1] J. M. Ziman, Models of Disorder, Cambridge Univ. Press, Cambridge, 1979.

[2] Р. Бэкстер, Точно решаемые модели в статистической механике, Мир, М., 1985.

[3] А.И. Гусев, Нестехиометрия, беспорядок, ближний и дальний порядок в твердом теле, Физматлит, М., 2007.

[4] М. Клеман, О.Д. Лаврентович, Основы физики частично упорядоченных сред, Физматлит, М., 2007.

[5] Н.Н. Медведев, Метод Вороного-Делоне в исследовании структуры некристаллических систем, Изд-во СО РАН, Новосибирск, 2000.

[6] A. V. Anikeenko, N. N. Medvedev, T. Aste, Phys. Rev. E, 77:3 (2008), 031101.

[7] D. Gratias, La Recherche, 178 (1986), 788-798.

[8] А. М. Братковский, Ю. А. Данилов, Г. И. Кузнецов, ФМM, 68:6 (1989), 1045-1095.

[9] В.В. Юдин, Стохастическая магнитная структура пленок с микропоровой системой, Наука, М., 1987.

[10] В. В. Юдин, Е. И. Рудик, А. В. Матохин и др., ФTT, 24:2 (1982), 443.

[11] В. В. Юдин, Т. А. Писаренко, Е. А. Любченко и др., Кристаллография, 44:3 (1999), 413.

[12] Е.А. Любченко, Древесные графы Кейли в исследовании сеточных структур мезодефектов квариевых стекол, Дис. ... канд. физ.-матем. наук, ДВГУ, Владивосток, 1999.

[13] В.В. Юдин, Т.А. Писаренко, Е.А. Любченко и др., Кристаллография, 47:2 (2002), $224-231$.

[14] Т.А. Писаренко, Фрактальность сеточных систем мезодефектов металлических и кварцевых стекол в спектральном и древесно-графовом представлениях, Дис. ... канд. физ.-матем. наук, ДВГУ, Владивосток, 2000.

[15] В. В. Юдин, Т. А. Писаренко, Е. А. Любченко, Информодинамика сетевых структур. Вероятность. Древесные графы. Фракталы, ДВГУ, Владивосток, 2003.

[16] В.В.Юдин, С. А. Щеголева, Т. А. Писаренко, ФТT, 43:11 (2001), 1991-1998.

[17] С. А. Щеголева, Т. А. Писаренко, В. В. Юдин, Поверхность, 12 (2001), 15-24.

[18] А. И. Олемской, И. В. Коплык, УФН, 165:10 (1995), 1105-1144.

[19] G. Paul, H. E. Stanley, Phys. Rev. E, 67:2 (2003), 026103; arXiv: cond-mat/0210345.

[20] T. Brookings, J. M. Carlson, J. Doyle, Phys. Rev. E, 72:5 (2005), 056120.

[21] J. L. Casti, Connectivity, Complexity, and Catastrophe in Large-Scale Systems, Internat. Ser. Appl. Systems Analys., 7, John Wiley \& Sons, New York, 1979.

[22] Н. Н. Моисеев, Математические задачи системного анализа, Наука, М., 1981.

[23] Г. Е. Шилов, Б. Л. Гуревич, Интеграл, мера и производная, Наука, М., 1967.

[24] Н. Мартин, Дж. Ингленд, Математическая теория энтропии, Мир, М., 1988. 
[25] М. А. Айзерман, Э. Р. Каянелло (ред.), Исследования по теории структур, Сборник научных трудов, Наука, М., 1988.

[26] F. Harari, E. M. Palmer, Graphical Enumeration, Academic Press, New York, 1973.

[27] А. И. Олемской, А. Я. Флат, УФН, 163:12 (1993), 1-50.

[28] H.-O. Peitgen, H. Jürgens, D. Saupe, Chaos and Fractals, Springer, New York, 2004.

[29] Я. Б. Зельдович, Д. Д. Соколов, УФН, 146:3 (1985), 493-506.

[30] В. В. Юдин, Т. А. Писаренко, Е. А. Любченко, О. А. Чуднова, Изв. РАН. Сер. физ., 65:10 (2001), 1405-1410.

[31] Д. А. Полянский, Теоретико-информационный анализ минимального класса квазикристаллических структур, Дис. ... канд. физ.-матем. наук, ДВГУ, Владивосток, 2005.

[32] В.В.Юдин, Ю. А. Карыгина, Кристаллография, 46:6 (2001), 1004-1008.

[33] В. М. Золотарев, Современная теория суммирования независимых случайных величин, Наука, М., 1986.

[34] В. В. Юдин, П. Л. Титов, А. Н. Михалюк, Изв. РАН. Сер. физ., 73:9 (2009), 1340-1347.

Поступила в редакцию 16.07.2009, после доработки 17.12.2009 